\title{
Title: Mutual Potentiation of Plant Immunity by Cell-surface and
} Intracellular Receptors

Intracellular Receptors

Authors: Bruno Pok Man Ngou ${ }^{1}$, Hee-Kyung Ahn', Pingtao Ding ${ }^{1,2 *}$, Jonathan DG Jones ${ }^{\text {* }}$

${ }^{1}$ The Sainsbury Laboratory, University of East Anglia, Norwich Research Park, Norwich NR4 $7 \mathrm{UH}, \mathrm{UK}$

${ }^{2}$ Current address: Institute of Biology Leiden, Leiden University, Sylviusweg 72, Leiden 2333

BE, The Netherlands

* Correspondence to: p.ding@biology.leidenuniv.nl; jonathan.jones@tsl.ac.uk

The plant immune system involves cell-surface receptors that detect intercellular pathogenderived molecules, and intracellular receptors that activate immunity upon detection of pathogen-secreted effectors that act inside the plant cell. Surface receptor-mediated immunity has been extensively studied ${ }^{1}$, but intracellular receptor-mediated immunity has rarely been investigated in the absence of surface receptor-mediated immunity. Furthermore, interactions between these two immune pathways are poorly understood. By activating intracellular receptors in the absence of surface receptor-mediated immunity, we dissected interactions between the two distinct immune systems. Recognition by surface receptors activates multiple protein kinases and NADPH oxidases; we find intracellular receptors primarily potentiate the activation of these proteins by elevating their abundance via multiple mechanisms. Reciprocally, the intracellular receptor-dependent hypersensitive response is strongly enhanced by activation of surface receptors. Activation of either immune system alone is insufficient to provide effective resistance against the bacterial pathogen Pseudomonas syringae. Thus, immune pathways activated by cell-surface and intracellular receptors mutually potentiate to activate strong defense that thwarts pathogens. These 
findings reshape our understanding of plant immunity and have broad implications for crop improvement.

\section{Main Text}

Plant cell-surface pattern-recognition receptors (PRRs) recognize pathogen-associated molecular patterns (PAMPs) and signal via plasma-membrane-associated co-receptor kinases, and intracellular protein kinases ${ }^{1}$. Ligand-dependent association between PRRs and these protein kinases activates calcium influx, production of reactive oxygen species (ROS) via activation of NADPH oxidases encoded by respiratory burst oxidase homolog (Rboh) genes, activation of mitogen-activated protein kinases (MAPKs) and induction of defense genes ${ }^{1}$.

Intracellular nucleotide-binding, leucine-rich-repeat-containing (NLR) receptors activate immune responses upon recognition of pathogen effectors. Plant sensor NLRs carry either an N-terminal coiled-coil (CC) domain, or an N-terminal Toll/Interleukin-1 receptor/Resistance protein (TIR) domain $^{2,3}$. Upon activation, the CC-NLR ZAR1 forms pentameric resistosome complexes, associates with plasma membranes (PMs) and likely perturbs their integrity ${ }^{4}$. The TIR-NLRs Roq1 and RPP1 form tetrameric resistosomes with effectors XopQ and ATR1, respectively, ${ }^{5,6}$ Upon activation, plant TIR-NLRs require NADase activity of their TIR domains to activate defense ${ }^{7}$. TIR-NLR signaling involves the lipase-like proteins EDS1, SAG101 and PAD4 ${ }^{8}$. PRRs activate pattern-triggered immunity (PTI), and NLRs effector-triggered immunity (ETI) ${ }^{9}$. How PTI and ETI interact to arrest pathogens is poorly understood.

\section{ETI enhances PTI defense responses}

To study ETI without PTI, we generated an Arabidopsis thaliana (Arabidopsis) line with estradiolinducible expression of bacterial effector AvrRps4 recognized by an intracellular TIR-NLR pair, RRS1 and RPS4 (RRS1/RPS4). Estradiol induces AvrRps4 expression and activates ETI ${ }^{\text {AvrRps4. }}$ Pre-activation of ETI ${ }^{\text {AvrRps4 }}$ elevates plant resistance against Pseudomonas syringae pv. tomato (Pst) DC3000 ${ }^{10}$. To test if ETI ${ }^{\text {AvrRps4 }}$ potentiates PTI, we measured ROS production triggered by flagellin-derived peptide flg22 (a bacterial PAMP) after pre-activating ETI ${ }^{\text {AvrRps4 }}$. ETI ${ }^{\text {AvrRps4 }}{ }^{\text {pre- }}$ activation elevates ROS production induced by flg22, but induction of ETI ${ }^{\text {AvrRps4 }}$ alone does not activate ROS production (Extended Fig 1a-b). Estradiol pre-treatment in an eds1-2 mutant 
background does not elevate flg22-induced ROS (Extended Fig 1c-d). Thus, ETI ${ }^{\text {AvrRps4 }}$ enhances, but does not initiate, PTI.

During bacterial infection, PTI activation precedes effector delivery. To mimic this, we treated plants with flg22, or estradiol, or "flg22 + estradiol", to activate PTI, or ETI ${ }^{\text {AvrRps4 }}$ or "PTI +

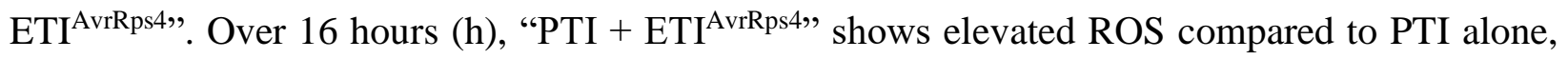
particularly during Phase III of the burst (Fig 1a, b and Extended Fig 1e, f). ETI ${ }^{\text {AvrRps4 }}$ enhances ROS production triggered by other PAMPs (elf18, C10:0, nlp20 and chitin) and the DAMP pep1 (Extended Fig 2). We investigated if ETI mediated by CC-NLRs also potentiates PTI. The CC-

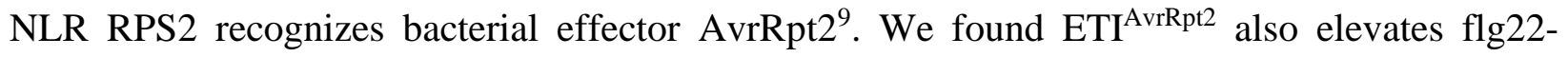
induced ROS (Extended Fig 1h-j). Thus, ETI activated by both TIR- or CC-NLRs can enhance ROS induced by PAMPs.

As "PTI + ETI" enhances the ROS burst of PTI alone, we assessed hydrogen peroxide $\left(\mathrm{H}_{2} \mathrm{O}_{2}\right)$

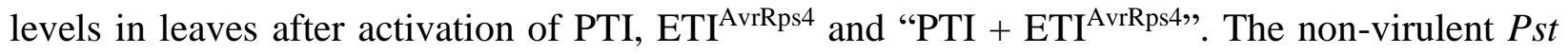
DC3000 $h r c C$ mutant $\left(h r c C^{-}\right)$induces PTI. Using diaminobenzidine (DAB) staining, "PTI + ETI $^{\text {AvrRps4", }}{ }^{\text {but not PTI or ETI }}{ }^{\text {AvrRps4 }}$ alone, trigger strong $\mathrm{H}_{2} \mathrm{O}_{2}$ accumulation after 2 days (Fig 1c). $\mathrm{H}_{2} \mathrm{O}_{2}$ promotes peroxidase-mediated cross-linking of proteins and phenolics in callose cell wall appositions during $\mathrm{PTI}^{11}$. ETI ${ }^{\text {AvrRps4 }}$ alone induces some callose deposition (Fig 1d). Callose

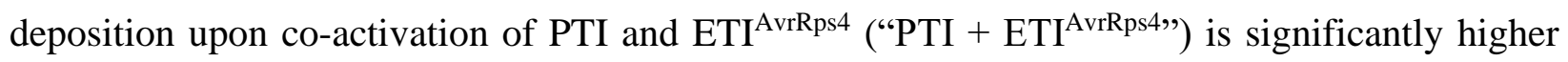
than the sum of that induced by PTI and ETI ${ }^{\text {AvrRps4 }}$ alone (Fig 1d-e). Thus, PTI and ETI together enhance callose deposition. Furthermore, the expression of PTI-responsive genes such as FRK1,

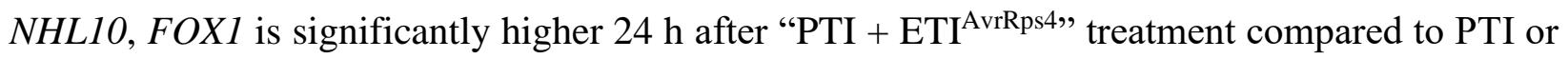
ETI $^{\text {AvrRps4 }}$ alone (Fig 1f and Extended Fig 1g). In summary, PTI-induced physiological changes are potentiated and enhanced by ETI.

Upon PAMP recognition, phosphorylation of the receptor-like cytoplasmic kinase subfamily VII (RLCK-VII) member BIK1 activates the NADPH oxidase RbohD via phosphorylation at its $39^{\text {th }}$ and $343^{\text {rd }}$ serine residues (S39 and S343). Activated RbohD produces extracellular ROS ${ }^{12,13}$. PTI also activates MAPKs, such as MPK3 and MPK6, contributing to transcriptional reprogramming (Extended Fig 3a $)^{14}$. We compared the activation of BIK1, RbohD and MAPKs during PTI and

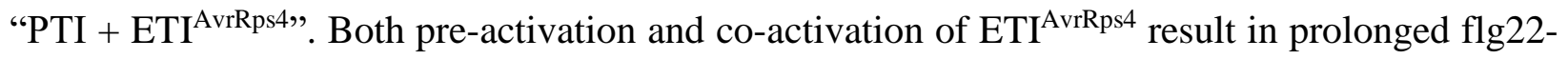


induced phosphorylation of BIK1, RbohD (at S39 and S343) and MPK3 (Figure 2a, b, Extended Fig 3b-e). However, ETI ${ }^{\text {AvrRps4 }}$ activation alone does not lead to phosphorylation of RbohD and MAPKs (Figure 2c, d) ${ }^{10}$. To investigate how ETI potentiates PTI, we monitored accumulation of

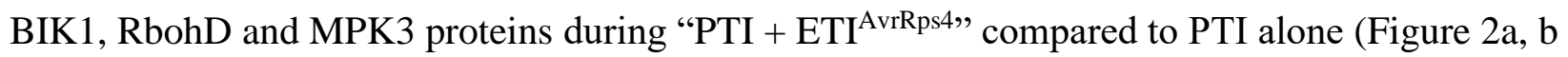

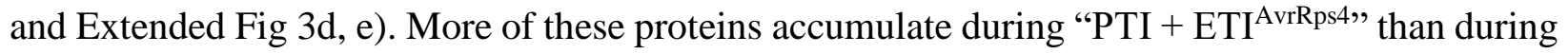
PTI alone. We assessed protein levels of multiple PTI signaling components during ETI activated in four additional inducible effector-expressing lines: AvrRpp4, AvrRpt2, AvrRpm1 and AvrPphB, which are recognized by TIR-NLR RPP4 and CC-NLRs RPS2, RPM1 and RPS5, respectively (Extended Fig 3f $)^{15}$. ETI triggered by these effectors elevates protein accumulation of BAK1, SOBIR1, BIK1, RbohD and MPK3 but not CERK1, FLS2, MPK4 and MPK6 (Extended Fig 3g).

Transcription and translation are strongly correlated during ETI ${ }^{16}$. We tested if PTI signaling components are elevated by transcriptional induction. ETI triggered by different effectors strongly elevates transcript abundance of $B A K 1, S O B I R 1, B I K 1, R b o h D$ and $M P K 3$, and weakly that of CERK1, FLS2, RbohF, MPK4 and MPK6 (Extended Fig 4a, b). Both protein and transcript

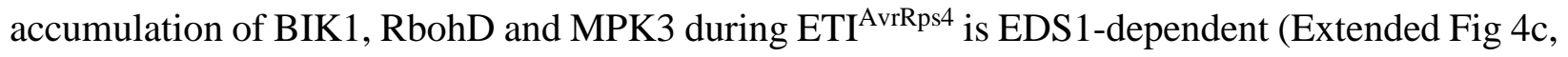
d). Thus, ETI alone boosts transcription of many genes involved in PTI signaling. We performed genome-wide expression profiling $4 \mathrm{~h}$ after induction of ETI ${ }^{\mathrm{AvrRps} 4}$ and found $\sim 10 \%$ of the transcriptome shows significant differential gene expression (Extended Fig 5a, b). Most upregulated genes are enriched in immunity-related biological processes, especially PRR signaling pathways (Fig 2c, Extended Fig 5c-e). Additional PTI signaling components such as EFR, PEPR1/2, LORE, LYK5, XLG2, CNGC19 and MKK4/5 are highly upregulated during ETI ${ }^{\mathrm{AvrRps} 4}$. Thus, ETI-dependent gene induction elevates the abundance of PTI signaling components.

Previous studies suggest substantial overlap between PTI- and "PTI + ETI"-induced transcriptional reprogramming ${ }^{17,18}$. We tested if increases in PTI signaling components during ETI are solely due to transcriptional activation. Transcript and protein levels of several PTI signaling components were monitored over a 24-h time-course post ETI ${ }^{\text {AvrRps4 }}{ }^{-i n d u c t i o n}$ (Fig 3a, b). Consistent with the protein level, SOBIRI and BAKl transcripts are highly induced by ETI ${ }^{\text {AvrRps } 4}$ (Fig 3a, b). However, BIK1, RbohD and MPK3 mRNAs are upregulated briefly and then downregulated after $3 \mathrm{~h}$, while increases in their protein levels are sustained over $24 \mathrm{~h}$ (Fig 3a, b and Extended Fig 6a-e). CERK1, MPK4 and MPK6 transcripts are weakly induced without 


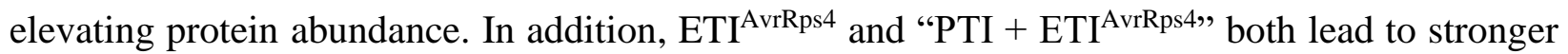
BIK1, RbohD and MPK3 accumulation compared to PTI, but their transcript levels differ only slightly between different conditions (Extended Fig 6f). These results imply that increases in PTIsignaling components during $\mathrm{ETI}^{\mathrm{AvrRps} 4}$ involves both transcriptional and post-transcriptional regulation.

We investigated accumulation of PTI-signaling components during ETI ${ }^{\mathrm{AvrRps} 4}$ using the translation inhibitor cycloheximide (CHX) and/or a proteasome inhibitor MG132. CHX blocks the accumulation of BIK1, RbohD, MPK3 and BAK1 during ETI, but not MPK6 or Actin (Fig 3c and Extended Fig 7a, b). MG132 treatment results in higher accumulation of BIK1 and RbohD but has no effect on MPK3 or BAK1 (Fig 3c and Extended Fig 7a, b). MPK3 accumulation is similar between the combined treatment of CHX and MG132 ("CHX + MG132") and CHX alone (Extended Fig 7b), suggesting that elevated MPK3 protein accumulation is likely due to increased translation rather than decreased protein degradation. BIK1 and RbohD protein levels increase with "CHX + MG132" treatment compared to those with $\mathrm{CHX}$ (Extended Fig 7b), implying that protein turnover of BIK1 and RbohD also plays a role ${ }^{19,20}$. However, this increase was not observed with FLS2, BAK1 or epitope-tagged RPS4 (Extended Fig 7c). Since translational reprogramming also contributes to immunity ${ }^{21}$, we compared abundance of ribosome-bound transcripts of ICS1, SOBIR1, BAK1, BIK1, RbohD and MPK3, normalized to a housekeeping gene EF1 $\alpha$ during mock and ETI ${ }^{\text {AvrRps4 }}$ treatment (Extended Fig 7d-f). ETI-induced increases in mRNA levels for BIK1, RbohD and MPK3 are matched by elevation in ribosome-loaded mRNA levels (Extended Fig 7gh). ETI thus elevates protein levels of PTI signaling components via multiple and distinct mechanisms that will be the subject of future investigations.

\section{ETI functions through PTI}

Whether ETI and PTI activate the same or distinct mechanisms is poorly defined, because ETI responses are rarely investigated in the absence of PTI. We tested whether (i) PTI provides the main defense mechanism against pathogens and (ii) ETI enhances PTI by replenishing PTI components, thus restoring effector-attenuated PTI.

We challenged plants with non-virulent Pst DC3000 $\mathrm{hrcC}^{-}$and found protein levels of BIK1 and RbohD are slightly elevated during PTI, and MAPKs are activated and show elevated 
phosphorylation. After infiltration with a virulent strain Pst DC3000, PTI-induced protein accumulation of BIK1 and RbohD, and MAPK activation is reduced compared to $h r c C^{-}$, consistent with effector-triggered susceptibility (ETS) ${ }^{9}$. We co-infiltrated plants with DC3000 and estradiol to co-induce ETI ${ }^{\text {AvrRps4 }}$ which restored protein levels of BIK1, RbohD and MPK3 and prolonged activation of MAPKs (Extended Fig 8a). This indicates that ETI overcomes ETS and restores PTI signaling capacity.

During natural infections, ETI is rarely activated without PTI. We hypothesized that ETI provides robust resistance by restoring and elevating the abundance of PTI signaling components, compensating for their turnover upon activation and attenuation by ETS (Extended Fig 8b). This model implies NLR-mediated resistance functions through PTI. We tested if PTI is required for NLR-dependent ETI-enhanced disease resistance by infiltrating the PTI-compromised mutants bak1-5 bkk1-1 and fls2 efr with Pst DC3000 delivering AvrRps4 (DC3000:AvrRps4) ${ }^{22}$. Remarkably, bakl-5 bkk1-1 is as susceptible as the NLR mutant rps4-2 rps $4 b$-2 that cannot detect AvrRps4 (Figure 4a and Extended Fig 8c), while fls2 efr also showed enhanced susceptibility to DC3000:AvrRps4 compared to wild type (Extended Fig 8d-g). These data show that PTI is required for RRS1/RPS4-dependent resistance to bacteria, and that activation of ETI in the absence of PTI is not sufficient for enhanced resistance against $P$. syringae in Arabidopsis. In addition, Yuan et al (eo-submitted 20200406411$)$ provide complementary data, independently showing that PTI is required for induced bacterial resistance mediated by multiple NLRs.

\section{PTI potentiates ETI-induced cell death}

ETI in the presence of PTI often culminates in hypersensitive cell death responses (HR). Arabidopsis infiltration with a non-pathogenic P. fluorescens Pf0-1 delivering AvrRps4 (Pf0-

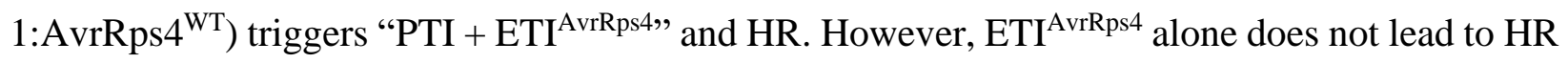
(Extended Fig 9a $)^{10}$. We used a Pf0-1 strain delivering a mutant allele of AvrRps4 (Pf01:AvrRps $4^{\text {mut }}$ ) to activate PTI. Co-activation of PTI and ETI ${ }^{\text {AvrRps4 }}$ results in HR and elevated electrolyte leakage (a widely used indicator of cell death), unlike PTI or ETI ${ }^{\text {AvrRps4 }}$ alone (Extended Fig 9a, b). To test if other PTI-inducers also potentiate HR, we repeated the experiment with either $h r c C^{-}$strain Pf0-1, a mixture of PAMPs and a DAMP (flg22, elf18 and pep1), or PAMPs or a DAMP alone (flg22, elf18, pep1, C10:0, nlp20 or chitin) to activate PTI ${ }^{1}$. In all cases, only PAMP 
infiltration combined with ETI ${ }^{\text {AvrRps4 }}$ triggers HR (Figure $4 \mathrm{~b}$ and Extended Fig 9c). Thus, PTI potentiates ETI-induced HR.

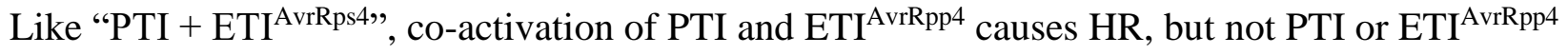
alone (Extended Fig 9e). In contrast, inducible expression of AvrRpt2, AvrRpm1 and AvrPphB that are recognized by CC-NLRs can trigger HR in the absence of PTI (Extended Fig 9d). By reducing levels of estradiol or dexamethasone, we defined sub-lethal levels of AvrRpt2, AvrRpm1 and AvrPphB induction. At these levels, CC-NLR mediated HR was also enhanced by PTI coactivation (Extended Fig 9e). Thus, PTI activation enhances HR triggered by multiple NLRs.

MAPKs and Rboh proteins promote ETI-dependent $\mathrm{HR}^{23,24}$. To understand PTI-enhanced ETIassociated HR, we investigated the role of MAPKs and Rbohs during ETI alone. We found MAPKs are phosphorylated during ETI ${ }^{\text {AvrRpm1 }}$, ETI $^{\text {AvrRpt2 }}$ and ETI ${ }^{\text {AvrPphB }}$, but not during ETI ${ }^{\text {AvrRps4 }}$

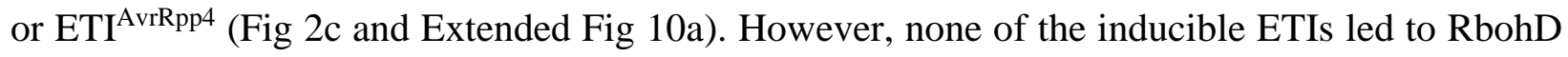

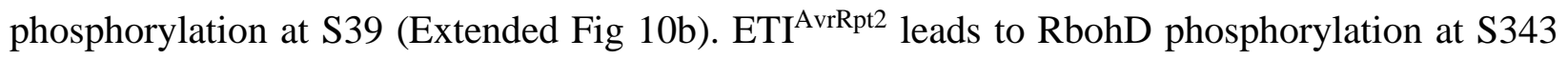
and $\mathrm{S} 347^{25}$, which might explain why ETI ${ }^{\text {AvrRpt2 }}$ activates a weak ROS burst (Extended Fig 1h-j).

Since ETI potentiates PTI-induced activation of MPK3 and RbohD, and ETI alone leads to weak or no activation of these components, we tested if HR enhancement by PTI involves the ETIpotentiated activity of MAPKs and NADPH oxidases. In an Arabidopsis line MPK6SR, an mpk3 mpk6 double mutant is complemented by a mutant MPK6 allele $\left(\mathrm{MPK}^{\mathrm{YG}}\right)^{26}$. Activity of MPK6 ${ }^{\mathrm{YG}}$ but not the wild-type MPK6 can be inhibited by an ATP analogue 1-NA-PP1 ${ }^{26}$. We tested the

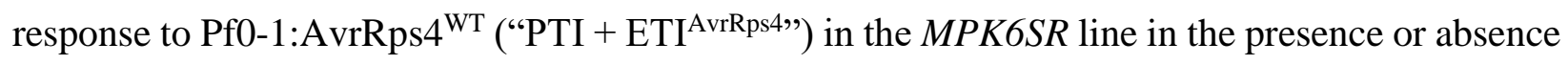
of 1-NA-PP1. Like others ${ }^{23}$, we found inhibition of MPK6 ${ }^{\mathrm{YG}}$ in MPK6SR prevents ETI ${ }^{\mathrm{AvrRps} 4}$ associated HR even in the presence of PTI (Extended Fig 10c). Furthermore, HR induced by Pf01:AvrRps $4^{\mathrm{WT}}$ is reduced in the NADPH oxidase mutant $r$ bohd rbohf (Extended Fig 10d). Together, these results demonstrate that the activation of MAPK and NADPH oxidases during "PTI + ETI $^{\text {AvrRps4" }}$ contributes to HR.

\section{Discussion}

We show here that ETI requires PTI to provide effective resistance. PTI can halt pathogens through nutrient restriction, cell wall fortification, suppression of bacterial type III secretion and induction of antimicrobial compounds ${ }^{11,27,28}$. ETI enhances PTI-induced defense responses via upregulation 
of PTI signaling components, and transcriptional, translational and/or protein turnover control (Extended Fig 10e). How this is achieved for each PTI component remains to be determined. We also show that the stronger immune response during "PTI + ETI" involves mutual potentiation of these two systems.

Our data, and those of Yuan et al (co-submitted manuscript, 2020-04-06411), support a model in which defenses activated by PRR-dependent signaling are the primary source of immunity, and activated NLR receptors act to replenish PRR signaling components and enhance PRR-dependent signaling, counteracting attenuation by turnover upon activation and by pathogen effectors (Fig 4c). In turn, PRR-mediated immunity can potentiate ETI outputs such as HR to further restrict pathogen proliferation. These data are highly relevant to elevating crop disease resistance. Many $N L R$ genes are semi-dominant, suggesting ETI strength is rate-limiting for resistance ${ }^{29}$. Thus, when PTI is present, stacks of multiple NLR genes should provide physiologically stronger resistance, as well as enhancing genetic durability, and are a potential source of non-host resistance ${ }^{30}$. Other reports have indicated synergistic functions of cell-surface and intracellular receptors in mammalian immunity ${ }^{31,32}$, highlighting the relevance of these insights to multiple host-pathogen systems.

\section{References}

1. Couto, D. \& Zipfel, C. Regulation of pattern recognition receptor signalling in plants. Nat. Rev. Immunol. 16, 537-552 (2016).

2. Jones, J. D. G., Vance, R. E. \& Dangl, J. L. Intracellular innate immune surveillance devices in plants and animals. Science 354, (2016).

3. Monteiro, F. \& Nishimura, M. T. Structural, functional, and genomic diversity of plant NLR proteins: an evolved resource for rational engineering of plant immunity. Annu. Rev. Phytopathol. 56, 243-267 (2018).

4. Wang, J. et al. Reconstitution and structure of a plant NLR resistosome conferring immunity. Science 364, (2019).

5. Martin, R. et al. Structure of the activated ROQ1 resistosome directly recognizing the pathogen effector XopQ. Science 370, eabd9993 (2020).

6. Ma, S. et al. Direct pathogen-induced assembly of an NLR immune receptor complex to form a holoenzyme. Science 370, eabe3069 (2020). 
7. Duxbury, Z., Wu, C. \& Ding, P. A Comparative Overview of the Intracellular Guardians of Plants and Animals: NLRs in Innate Immunity and Beyond. Annu. Rev. Plant Biol. (2021). at <https://doi.org/10.1146/annurev-arplant-080620-104948>

8. Lapin, D. et al. A Coevolved EDS1-SAG101-NRG1 Module Mediates Cell Death Signaling by TIR-Domain Immune Receptors. Plant Cell 31, 2430-2455 (2019).

9. Jones, J. D. G. \& Dangl, J. L. The plant immune system. Nature 444, 323-329 (2006).

10. Ngou, B. P. M. et al. Estradiol-inducible AvrRps4 expression reveals distinct properties of TIR-NLR-mediated effector-triggered immunity. J. Exp. Bot. 71, 2186-2197 (2020).

11. Voigt, C. A. Callose-mediated resistance to pathogenic intruders in plant defense-related papillae. Front. Plant Sci. 5, 168 (2014).

12. Kadota, Y. et al. Direct regulation of the NADPH oxidase RBOHD by the PRR-associated kinase BIK1 during plant immunity. Mol. Cell 54, 43-55 (2014).

13. Li, L. et al. The FLS2-associated kinase BIK1 directly phosphorylates the NADPH oxidase RbohD to control plant immunity. Cell Host Microbe 15, 329-338 (2014).

14. Meng, X. \& Zhang, S. MAPK cascades in plant disease resistance signaling. Annu. Rev. Phytopathol. 51, 245-266 (2013).

15. Kourelis, J. \& van der Hoorn, R. A. L. Defended to the nines: 25 years of resistance gene cloning identifies nine mechanisms for R protein function. Plant Cell 30, 285-299 (2018).

16. Meteignier, L.-V. et al. Translatome analysis of an NB-LRR immune response identifies important contributors to plant immunity in Arabidopsis. J. Exp. Bot. 68, 2333-2344 (2017).

17. Ding, P. et al. High-resolution expression profiling of selected gene sets during plant immune activation. Plant Biotechnol. J. (2020). doi:10.1111/pbi.13327

18. Ding, P. et al. Chromatin accessibility landscapes activated by cell surface and intracellular immune receptors. BioRxiv (2020). doi:10.1101/2020.06.17.157040

19. Ma, X. et al. Ligand-induced monoubiquitination of BIK1 regulates plant immunity. Nature 581, 199-203 (2020).

20. Lee, D. et al. Regulation of reactive oxygen species during plant immunity through phosphorylation and ubiquitination of RBOHD. Nat. Commun. 11, 1838 (2020).

21. Urquidi Camacho, R. A., Lokdarshi, A. \& von Arnim, A. G. Translational gene regulation in plants: A green new deal. Wiley Interdiscip Rev RNA 11, e1597 (2020). 
22. Roux, M. et al. The Arabidopsis leucine-rich repeat receptor-like kinases BAK1/SERK3 and BKK1/SERK4 are required for innate immunity to hemibiotrophic and biotrophic pathogens. Plant Cell 23, 2440-2455 (2011).

23. Su, J. et al. Active photosynthetic inhibition mediated by MPK3/MPK6 is critical to effectortriggered immunity. PLoS Biol. 16, e2004122 (2018).

24. Torres, M. A., Dangl, J. L. \& Jones, J. D. G. Arabidopsis gp91phox homologues AtrbohD and AtrbohF are required for accumulation of reactive oxygen intermediates in the plant defense response. Proc. Natl. Acad. Sci. USA 99, 517-522 (2002).

25. Kadota, Y. et al. Quantitative phosphoproteomic analysis reveals common regulatory mechanisms between effector- and PAMP-triggered immunity in plants. New Phytol. 221, 2160-2175 (2019).

26. Xu, J. et al. A chemical genetic approach demonstrates that MPK3/MPK6 activation and NADPH oxidase-mediated oxidative burst are two independent signaling events in plant immunity. Plant J. 77, 222-234 (2014).

27. Yamada, K., Saijo, Y., Nakagami, H. \& Takano, Y. Regulation of sugar transporter activity for antibacterial defense in Arabidopsis. Science 354, 1427-1430 (2016).

28. Anderson, J. C. et al. Decreased abundance of type III secretion system-inducing signals in Arabidopsis mkp1 enhances resistance against Pseudomonas syringae. Proc. Natl. Acad. Sci. USA 111, 6846-6851 (2014).

29. Jones, J. D. Putting knowledge of plant disease resistance genes to work. Curr. Opin. Plant Biol. 4, 281-287 (2001).

30. Cevik, V. et al. Transgressive segregation reveals mechanisms of Arabidopsis immunity to Brassica-infecting races of white rust (Albugo candida). Proc. Natl. Acad. Sci. USA 116, 2767-2773 (2019).

31. Kelley, N., Jeltema, D., Duan, Y. \& He, Y. The NLRP3 inflammasome: an overview of mechanisms of activation and regulation. Int. J. Mol. Sci. 20, (2019).

32. Wolf, A. J. \& Underhill, D. M. Peptidoglycan recognition by the innate immune system. Nat. Rev. Immunol. 18, 243-254 (2018). 


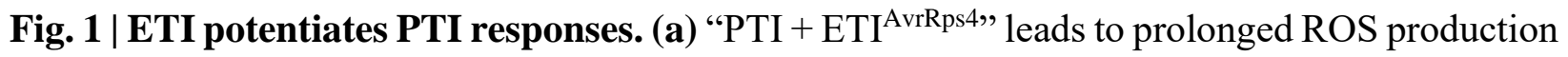
from 300-960 mins (Phase III). Solid line represents mean \pm standard error of the mean (S.E.;

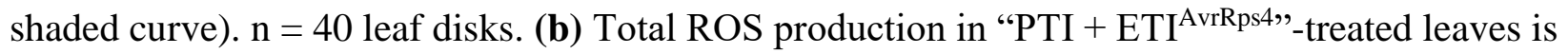
significantly higher than PTI-treated leaves. $n=120$ leaf disks from three independent

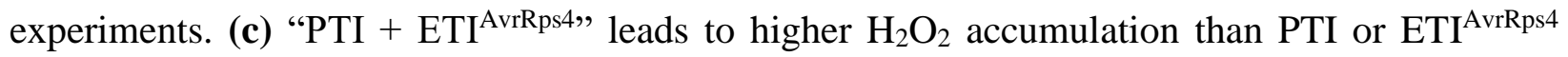

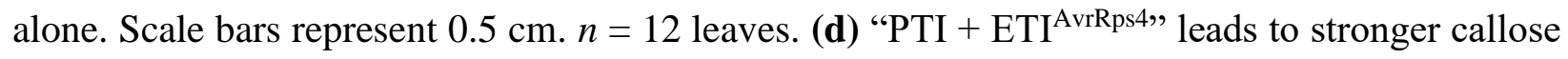
deposition than PTI or ETI ${ }^{\text {AvrRps4 }}$ alone. Numbers represent the mean \pm S.E. (e) Callose deposition

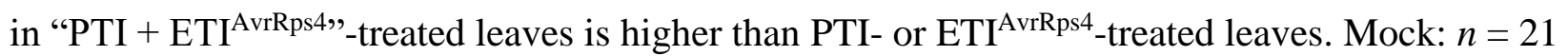
leaves; PTI, ETI, "PTI + ETI": $n=23$ leaves. (b, e) Centre lines represent medians; bounds of box indicate the $25^{\text {th }}$ and $75^{\text {th }}$ percentiles; whiskers represent $1.5 \times$ interquartile range from $25^{\text {th }}$ and $75^{\text {th }}$ percentiles. Data points from 3 biological replicates were analyzed with one-sided Kruskal-Wallis test with Holm correction, then followed by post hoc Dunn's test. Data points with different letters indicate significant differences of $\mathrm{P}<0.05$. P-values were adjusted with Holm correction, and

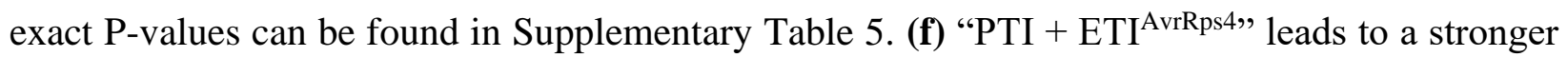
FRK1, NHL10, FOX1 transcript accumulation compared to PTI or ETI ${ }^{\mathrm{AvrRps} 4}$ alone. Data points from 3 independent experiments were plotted onto the graphs, with \pm S.E. for error bars. Two-sided

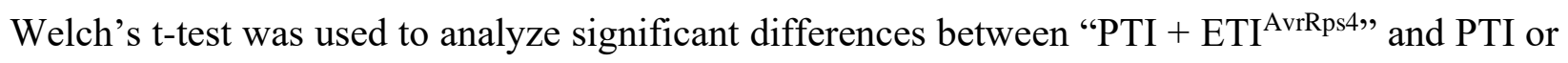
ETI $^{\text {AvrRps4 }}{ }^{*}\left({ }^{*}, \mathrm{P} \leq 0.05 ; * *, \mathrm{P} \leq 0.01 ; * * *, \mathrm{P} \leq 0.005 ; * * * *, \mathrm{P} \leq 0.001\right.$; otherwise, not significant). Exact P-values can be found in Supplementary Table 5. All experiments were repeated at least three times with similar results.

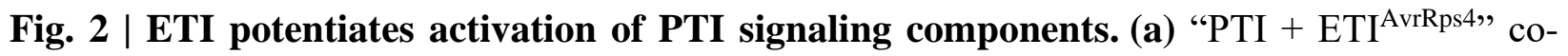
activation leads to increased MPK3 accumulation and prolonged phosphorylation compared to

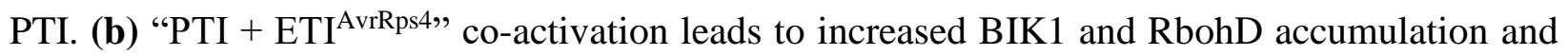
prolonged phosphorylation compared to PTI. (c) ETI ${ }^{\text {AvrRps4 }}$ activation alone does not trigger RbohD-S39 phosphorylation. (d) ETI ${ }^{\text {AvrRps4 }}$ alone does not lead to MAPK activation. For (b, c), microsomal fractions from the samples were isolated for immunoblotting. Molecular weight marker (in $\mathrm{kDa}$ ) is indicated on the left. Ponceau staining (PS) was used as loading control. (e) RNA-seq results of the upregulation of PTI signaling pathway during ETI ${ }^{\text {AvrRps4 }}$. Heatmap representing the expression level of PTI signaling pathway genes, salicylic acid (SA) and pipecolic acid (PIP) biosynthesis pathway genes and photosynthetic pathway genes at $4 \mathrm{~h}$ after ETI ${ }^{\text {AvrRps } 4}$ 
induction. Red represents upregulation and blue represents downregulation. All experiments were repeated at least three times with similar results.

Fig. 3 | Accumulation of PTI signaling components during ETI. (a) Relative mRNA expression changes of SOBIR1, BAK1 (top panel), BIK1, RbohD, MPK3 (middle panel), and CERK1, MPK4,

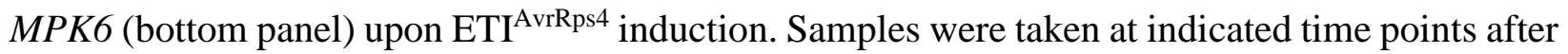
ETI $^{\text {AvrRps4 }}$ activation. All samples were normalized against expression of the corresponding genes in untreated samples $\left(\log _{2} \mathrm{FC}=0\right.$, dotted line). Solid line represents mean \pm S.E. (shaded band). (b) Protein accumulation of Actin, SOBIR1, BAK1, BAK1, RbohD, MPK3, CERK1, MPK4 and MPK6 at different time points; Actin is the loading control. Molecular weight is indicated on the left. Ponceau staining (PS) was used as additional loading control and shown in Extended Fig 6d. (c) Translation is necessary for the increased protein accumulation of MPK3, RbohD, BIK1, but not MPK6 and Actin. 7-day-old seedlings were pre-activated with ETI ${ }^{\text {AvrRps4 }}$ for $3 \mathrm{~h}$ and subsequently treated with cycloheximide $(50 \mu \mathrm{M}$; CHX), MG132 $(10 \mu \mathrm{M})$, or both for indicated times $(2,4,8 \mathrm{~h}$ ). Actin is loading control. Molecular weight (in $\mathrm{kDa}$ ) is indicated on the left. Ponceau staining (PS) images of corresponding blots are also shown. All experiments were repeated at least three times with similar results.

Fig. 4 | PTI and ETI function synergistically to provide robust immunity. (a) Both PTI and $\mathrm{ETI}^{\mathrm{AvrRps} 4}$ are required to provide effective immunity against $P$. syringae. Col-0, rps4-2 rps $4 b-2$ and bak1-5 bkk1-1 were infected with P. syringae pv. tomato (Pst) strain DC3000 carrying empty vector (grey) or AvrRps4 (pink). Both rps4-2 rps4b-2 (no ETI) and bakl-5 bkk1-1 (PTI-reduced) are insufficient to provide resistance against Pst DC3000 carrying AvrRps4 compared to Col-0 ("PTI + ETI"). $n=18$ leaves. Centre lines represent medians; bounds of box indicate the $25^{\text {th }}$ and $75^{\text {th }}$ percentiles; whiskers represent $1.5 \times$ interquartile range from $25^{\text {th }}$ and $75^{\text {th }}$ percentiles. Data points from 3 biological replicates were analyzed with one-way ANOVA, then followed by post hoc Tukey's HSD test. Data points with different letters indicate significant differences of $\mathrm{P}<$ 0.05. P-values were adjusted with Holm correction, and exact P-values can be found in Supplementary Table 5. (b) ETI ${ }^{\text {AvrRps4 }}$ leads to macroscopic HR only in the presence of PTI, activated by either non-virulent Pst DC3000 hrc $C^{-}$, P. fluorescens Pf0-1 or mixture of flg22, elf18 and pep1 (PAMPs). $n=18$ leaves. (c) Schematic representation of the plant immune system. PAMPs from pathogens are recognized by plant PRRs and induce PTI (red). Virulent pathogens 
secrete effectors to suppress PTI (green). Effectors are recognized by NLRs and induce ETI (dark yellow arrow), which potentiates PTI to produce robust immune response (blue arrow). All experiments were repeated at least three times with similar results.

\section{Methods}

Plant material and growth conditions

Arabidopsis thaliana Columbia-0 (Col-0) was used as wild type in this study. Seeds were sown on compost and plants were grown at $21{ }^{\circ} \mathrm{C}$ with $10 \mathrm{~h}$ under light and $14 \mathrm{~h}$ in dark, and at $70 \%$ humidity. The light level is approximately $180-200 \mu \mathrm{mol}$ with fluorescent tubes. Information about all plant materials can be found in the referred literatures ${ }^{26,33-37}$, and were kindly provided by Jeffery Dangl (Department of Biology, The University of North Carolina at Chapel Hill), Roger Innes (Department of Biology, Indiana University), Shuta Asai (RIKEN, Japan), Shuqun Zhang (Division of Biochemistry, University of Missouri), Xiufang Xin (Shanghai Institutes for Biology Sciences, Chinese Academy of Sciences) and Cyril Zipfel (The Sainsbury Laboratory, UK).

\section{$\underline{\text { ROS burst assay (pre-treatment with ETI) }}$}

Leaf discs harvested with a 6-mm-diameter cork borer from 5-week-old plants were placed in 96well plates with $200 \mu \mathrm{l}$ of deionized water overnight in dark (with abaxial surface of the leaves face down). Leaf discs were then soaked in mock solution (1\% DMSO) or $50 \mu \mathrm{M}$ est (estradiol to trigger ETI ${ }^{\text {AvrRps4}}$ ) for 6 h. $200 \mu \mathrm{l}$ of $20 \mathrm{~mm}$ luminol (Sigma-Aldrich, A8511), $0.02 \mathrm{mg} / \mathrm{ml}$ horseradish peroxidase (Sigma-Aldrich, P6782) and $100 \mathrm{nM}$ flg22 were added in each well. ROS production was measured with a Photek camera (East Sussex, UK). Data from each treatment is represented by 40 leaf discs in one biological replicate. Every plate was measured over 55 mins.

\section{$\underline{\text { ROS burst assay (co-treatment with ETI) }}$}

Leaf discs harvested with a 6-mm-diameter cork borer from 5-week-old plants were placed in 96well plates with $200 \mu \mathrm{l}$ of deionized water overnight in dark (with abaxial surface of the leaves face down). $200 \mu \mathrm{l}$ of $20 \mathrm{~mm}$ luminol (Sigma-Aldrich, A8511), $0.02 \mathrm{mg} / \mathrm{ml}$ horseradish peroxidase (Sigma-Aldrich, P6782) and indicated elicitors (concentration indicated in Supplementary Table 3) were added in each well. ROS production was measured with a Photek camera (East Sussex, UK). Data from each treatment is represented by 40 leaf discs in one biological replicate. Every plate was measured over the $16 \mathrm{~h}$. 
DAB staining

3,3'-diaminobenzidine (Sigma-Aldrich, D8001) was dissolved in water (1 mg/ml) and the $\mathrm{pH}$ is adjusted to 6 with sodium hydroxide. Arabidopsis leaves were infiltrated with indicated solutions (concentration indicated in Supplementary Table 3). Two days after infiltration, leaves were vacuum infiltrated with DAB solution for 30 mins and incubated in room temperature for $2 \mathrm{~h}$. The DAB solution was replaced with $100 \%$ ethanol and then boiled for 1 mins. The leaves are then further de-stained with $70 \%$ ethanol under room temperature. De-stained leaves were then scanned with EPSON Perfection V600 Photo. Scale bar $=0.5 \mathrm{~cm}$.

\section{Callose quantification}

Leaves from 5-week-old Arabidopsis were hand-infiltrated with the indicated solutions (concentration indicated in Supplementary Table 3) and covered for $24 \mathrm{~h}$. Leaves were then handinfiltrated with $1 \times$ PBS buffer containing $0.01 \%$ Aniline Blue. Leaf discs were then harvested with a 6-mm-diameter cork borer for imaging. Images were taken by an epifluorescence microscope with UV filter (excitation, 365/10 nm; emission, 460/50 nm). The number of callose dots was calculated by ImageJ software. One leaf disc was harvested per leaf. At least 6 leaves from individual plants were included per treatment in one biological replicate.

Gene expression analysis by reverse transcription-quantitative polymerase chain reaction (RT$\underline{\mathrm{qPCR}})$

Arabidopsis thaliana tissues were treated with indicated solutions (concentration indicated in Supplementary Table 3 ) for indicated time point. Tissues were then snap-frozen and RNA was isolated by RNeasy Plant Mini Kit (74904; Qiagen) and used for subsequent RT-qPCR analysis. Reverse transcription was carried out with SuperScript IV Reverse Transcriptase (18090050; ThermoFisher Scientific). qPCR was performed with KAPA SYBR® FAST (Roche) using the CFX96 Touch ${ }^{\mathrm{TM}}$ Real-Time PCR Detection System. Primers for qPCR analysis are listed in Supplementary Information Table 2. Data were analyzed using the double delta $\mathrm{Ct}$ method ${ }^{38}$.

$\underline{\text { Immunoblotting (pre-treatment with ETI) }}$

5-week-old Est:AvrRps4 leaves were sprayed with either mock or $50 \mu \mathrm{M}$ est solution (in $0.01 \%$ Silwet L-77) and covered for $6 \mathrm{~h}$. Leaves were then infiltrated with $100 \mathrm{nM}$ flg22. Samples were collected at indicated time points and snap-frozen in liquid nitrogen. Samples were lysed and 
proteins were extracted using GTEN buffer (10\% glycerol, $25 \mathrm{mM}$ Tris $\mathrm{pH}$ 7.5, $1 \mathrm{mM}$ EDTA, 150 $\mathrm{mM} \mathrm{NaCl}$ ) with $10 \mathrm{mM}$ DTT, $1 \% \mathrm{NP}-40$ and protease inhibitor cocktail (cOmplete ${ }^{\mathrm{TM}}$, EDTA-free; Merck), phosphatase inhibitor cocktail 2 (Sigma-Aldrich; P5726) and phosphatase inhibitor cocktail 3 (Sigma-Aldrich; P0044). After centrifugation at 13,000× rpm for 10 mins to remove cell debris, protein concentration of each sample was measured using the Bradford assay (Protein Assay Dye Reagent Concentrate; Bio-Rad). After normalization, extracts were incubated with $2 \times$ TruPAGETM LDS Sample Buffer (Sigma-Aldrich) at $70{ }^{\circ} \mathrm{C}$ for 10 mins. SDS-PAGE gels of different percentages were used to run protein samples of difference sizes. After transferring proteins from gels to PVDF membranes (Merck-Millipore) using Trans-Blot Turbo System (BioRad), membranes were blocked with 5\% nonfat dried milk in TBST for 1h, immunoblotted with antibodies specified in Supplementary Information Table 1. Anti-Rabbit IgG (whole molecule)Peroxidase antibody produced in goat (A0545; Merck-Sigma-Aldrich) was used as secondary antibody following the use of above antibodies. Ponceau S solution (P7170; Sigma-Aldrich) was used to stain the PVDF membrane for loading control. For RbohD and BIK1, plasma membrane protein was extracted for immunoblotting (see below).

\section{Immunoblotting (co-treatment with ETI)}

5-week-old est:AvrRps4 leaves were infiltrated with indicated solutions (concentration indicated in Supplementary Table 3) for indicated time point. Tissues were then collected and snap-frozen. Proteins were extracted and immunoblotting was performed as stated above. Concentrations of primary antibodies are specified in Supplementary Information Table 1. Anti-Rabbit IgG (whole molecule)-Peroxidase antibody produced in goat (A0545; Merck-Sigma-Aldrich) was used as secondary antibody following the use of above antibodies. Ponceau S solution (P7170; SigmaAldrich) was used to stain the PVDF membrane for loading control. For RbohD and BIK1, plasma membrane protein was extracted for immunoblotting (see below).

Plasma membrane protein extraction

Minute $^{\text {TM }}$ Plant Plasma Membrane Protein Isolation Kit (Invent Biotechnologies, SM-005-P) was used to extract total membrane fraction from Arabidopsis samples as instructed. Protein concentration of the cytosolic fraction from each sample was measured using the Bradford assay (Protein Assay Dye Reagent Concentrate; Bio-Rad). After normalization, total membrane fractions were dissolved in $2 \times$ TruPAGETM $^{\mathrm{TM}}$ LS Sample Buffer (Sigma-Aldrich) at $70{ }^{\circ} \mathrm{C}$ for 5 mins (in a 
minimal volume of $80 \mu \mathrm{l}$ ). $6 \%$ SDS-PAGE gels were used to run the protein samples. After transferring proteins from gels to PVDF membranes (Merck-Millipore) using Trans-Blot Turbo System (Bio-Rad), membranes were blocked with 5\% nonfat dried milk in TBST for $1 \mathrm{~h}$, immunoblotted with either BIK1, pS39-RbohD or pS343-RbohD antibodies kindly provided by Jian-Min Zhou (Institute of Genetics and Developmental Biology, Chinese Academy of Sciences) $)^{13}$. Concentrations of primary antibodies are specified in Supplementary Information Table 1. Anti-Rabbit IgG (whole molecule)-Peroxidase antibody produced in goat (A0545; Merck-Sigma-Aldrich) was used as secondary antibody. Ponceau S solution (P7170; SigmaAldrich) was used to stain the PVDF membrane for loading control.

\section{Immunoblotting}

5-week-old Arabidopsis thaliana leaves were treated with indicated solution (concentration indicated in Supplementary Table 3). Tissues were then collected and snap-frozen. Proteins were extracted and immunoblotting was performed as stated above. Concentrations of primary antibodies are specified in Supplementary Information Table 1. Anti-Rabbit IgG (whole molecule)-Peroxidase antibody produced in goat (A0545; Merck-Sigma-Aldrich) was used as secondary antibody following the use of above antibodies. Ponceau S solution (P7170; SigmaAldrich) was used to stain the PVDF membrane for loading control.

\section{$\underline{\text { RNA-seq and data analysis }}$}

Leaves from 5-week-old Arabidopsis estradiol-inducible AvrRps4 (est:AvrRps4) or est:AvrRps $4^{\text {mut }}{ }^{10}$ were hand-infiltrated with $50 \mu \mathrm{M}$ estradiol for 0 or $4 \mathrm{~h}$. Samples were collected and total RNA was isolated with TRI Reagent ${ }^{\circledR}$ (T9424: Sigma-Aldrich) and RNA Clean \& Concentrator-25 Kit (R1018; Zymo Research). RNA samples are processed by BGI and libraries are sequenced with BGISEQ-500 sequencing platform. At least $10 \mathrm{M}$ single-end 50-bp reads are obtained for each RNA-seq library. Adaptor-trimmed clean reads have been uploaded to the European Nucleotide Archive (ENA) (accession ID: PRJEB34955). After FastQC, Kallisto was used to map and quantify RNA-seq reads ${ }^{39}$, and kallisto_quant output files are submitted to the 3D RNA-seq tool for statistics and data visualization ${ }^{40}$. P-values for differentially expressed (DE) genes were generated with Fisher Z-transformation after Student's $t$-test and were adjusted with Benjamini and Hochberg's (BH) method ${ }^{40}$. 
$\underline{\text { Serial dilution to estimate protein abundance }}$

Fold changes of BIK1, RbohD and MPK3 protein accumulation upon ETI ${ }^{\text {AvrRps4 }}$ is estimated by serial dilution. Protein samples of ETI ${ }^{\text {AvrRps4 }}$ at 8 h were diluted $2 \times(1 / 2), 4 \times(1 / 4), 8 \times(1 / 8), 16 \times$ $(1 / 16)$ and $32 \times(1 / 32)$ in $2 \times$ TruPAGETM LDS Sample Buffer (Sigma-Aldrich). Samples were then loaded together with protein samples of ETI ${ }^{\mathrm{AvrRps} 4}$ at $0 \mathrm{~h}$ and ran on 10\% SDS-PAGE gels. After transferring the proteins from gels to PVDF membranes (Merck-Millipore) using Trans-Blot Turbo System (Bio-Rad), membranes were blocked with 5\% nonfat dried milk in TBST for $1 \mathrm{~h}$, immunoblotted with antibodies specified in Supplementary Information Table 1. Anti-Rabbit IgG (whole molecule)-Peroxidase antibody produced in goat (A0545; Merck-Sigma-Aldrich) was used as secondary antibody. Ponceau S solution (P7170; Sigma-Aldrich) was used to stain the PVDF membrane for loading control.

\section{Cycloheximide and MG132 treatment}

1-week-old seedlings of est:AvrRps4 Arabidopsis transgenic line were grown in liquid MS supplemented with $1 \%$ sucrose were pre-treated with $50 \mu \mathrm{M}$ estradiol or mock (DMSO) for $3 \mathrm{~h}$. After pre-treatment, cycloheximide (CHX; $50 \mu \mathrm{M})$, MG132 $(10 \mu \mathrm{M})$, or combination of CHX and MG132 were treated to seedlings in addition to estradiol or mock. Seedlings were harvested $2 \mathrm{~h}$, $4 \mathrm{~h}$, and $8 \mathrm{~h}$ after inhibitor treatments. Upon protein extraction, protein concentration was measured using Bradford assay, and protein samples were analyzed by immunoblotting as described above.

\section{Enrichment of ribosome}

Enrichment of ribosome was performed based on previous publications ${ }^{41,42}$ with modifications. 5week old Arabidopsis leaves of est:AvrRps4 were infiltrated with mock (1\% DMSO) or $50 \mu \mathrm{M}$ est for 6 h. $0.6 \mathrm{~g}$ of leaves were harvested and ground in liquid nitrogen and extracted with $5 \mathrm{ml}$ extraction buffer (0.2 M Tris-HCl, pH 8.4, $50 \mathrm{mM} \mathrm{KCl,} 25 \mathrm{mM} \mathrm{MgCl}_{2}, 0.5 \%$ or Nonidet P-40, 50 $\mu \mathrm{g} / \mathrm{ml}$ cycloheximide, RNase inhibitor (RNasin®, Promega). After centrifugation at 13,000× rpm for 10 mins, supernatant was loaded onto a 1.6 M sucrose cushion. Samples were ultracentrifuged at $170,000 \times \mathrm{g}$ for $16 \mathrm{~h}$. Pellet samples were resuspended in $1 \mathrm{ml}$ DEPC-treated water, and $800 \mu \mathrm{l}$ was used for RNA extraction and qPCR analysis and $200 \mu \mathrm{l}$ for protein extraction as described above.

Bacterial growth assay 
Pseudomonas syringae pv. tomato strain DC3000 carrying AvrRps4 or empty vector pVSP61 was grown on selective King's B (KB) medium plates for $48 \mathrm{~h}$ at $28{ }^{\circ} \mathrm{C}$. Bacteria were resuspended and the concentration was adjusted to 0.001 at $\mathrm{OD}_{600}$. Abaxial surfaces of 5-week-old Arabidopsis leaves were infiltrated with bacterial solution by a 1-ml needleless syringe. For quantification, two leaf discs per leaf were harvested with a 6-mm diameter cork borer (with disc area of $0.283 \mathrm{~cm}^{2}$ ). For "day 0", samples were ground in infiltration buffer $\left(10 \mathrm{mM} \mathrm{MgCl}_{2}\right)$ and spotted $(10 \mu \mathrm{l} / \mathrm{spot})$ on selective KB medium. For "day 3", samples were ground in infiltration buffer, serially diluted (into 5, 50, 500, 5,000, and 50,000 times), and spotted (6 $\mu \mathrm{l} / \mathrm{spot})$ on selective $\mathrm{KB}$ medium. The number of colonies (CFU per drop) was calculated, and bacterial growth was represented as CFU $\mathrm{cm}^{-2}$ of leaf tissue.

\section{$\underline{\text { HR assay in Arabidopsis }}$}

Pseudomonas fluorescens Pf0-1 engineered with a type III secretion system (PfO-1 "EtHAn" strains) expressing effectors, AvrRps4, AvrRps $4^{\text {KRVY135-138AAAA }}$ (mutant AvrRps4; AvrRps4 ${ }^{\text {mut }}{ }^{43}$, or pVSP61 empty vector were grown on selective $\mathrm{KB}$ plates for $24 \mathrm{~h}$ at $28{ }^{\circ} \mathrm{C}$. Wild-type Pseudomonas fluorescens were grown on KB plates with chloramphenicol for $24 \mathrm{~h}$ at $28{ }^{\circ} \mathrm{C}$. Pseudomonas syringae pv. tomato strain DC3000 hrc $C^{-}$or DC3000 were grown on KB plates with kanamycin for $48 \mathrm{~h}$ at $28{ }^{\circ} \mathrm{C}$. Bacteria were harvested from the plates, resuspended in infiltration buffer $\left(10 \mathrm{mM} \mathrm{MgCl}_{2}\right)$ and the concentration was adjusted to indicated $\mathrm{OD}_{600}$ (Supplementary Information Table 3). The abaxial surfaces of 5-week-old Arabidopsis leaves were hand infiltrated with indicted solution by a 1-ml needleless syringe. Cell death was monitored at indicated time points after infiltration.

\section{Electrolyte leakage assay}

5-week-old Arabidopsis leaves were infiltrated with indicated solutions (Supplementary Information Table 3) with a 1-ml needleless syringe. Leaf discs were collected with a 2.4-mmdiameter cork borer from infiltrated leaves. Discs were dried and washed in deionized water for $1 \mathrm{~h}$ before being floated on $10 \mathrm{ml}$ deionized water ( 15 discs per sample, three samples per biological replicate). Electrolyte leakage was measured as water conductivity with a Pocket Water Quality Meters (LAQUAtwin-EC-33; Horiba) at the indicated time points.

$\underline{\text { Statistical data analysis }}$ 
Statistical data were analyzed using the R software (https://www.r-project.org/), and the data were plotted using the Origin software. For statistical analysis, all data were tested for homoscedasticity with Levene's test, and normal distribution with Shapiro-Wilk test, and either parametric one-way ANOVA analysis followed by Tukey's post-hoc HSD test, or non-parametric Kruskal-Wallis test followed by Dunn's test were applied for statistical significance. Data points with different letters indicate significant differences of $\mathrm{P}<0.01$ for Tukey's HSD test results, and $\mathrm{P}<0.05$ for Dunn's test. Data points are plotted onto the graph, and number of samples for each data are indicated in corresponding figure legends. Three biological replicates were tested, and individual biological replicates are indicated with different shapes of the data points. qPCR assay results were analyzed using two-sided Welch's t-test for statistical significance (*, $\mathrm{P} \leq 0.05 ; * *, \mathrm{P} \leq 0.01 ; * * *, \mathrm{P} \leq 0.001$; $* * * *, \mathrm{P} \leq 0.0001$; otherwise, not significant) between samples. Detailed information of sample number, statistical analysis values for all experiments can be found in the Supplementary Table 5.

\section{Generation of schematic figures}

Schematic figures in Fig 2e, 4c, Extended Data Fig 3b, c, 5a, 7d and 10e were created with BioRender.com.

\section{$\underline{\text { References for Methods }}$}

33. Schwessinger, B. et al. Phosphorylation-dependent differential regulation of plant growth, cell death, and innate immunity by the regulatory receptor-like kinase BAK1. PLoS Genet. 7, e1002046 (2011).

34. Asai, S. et al. A downy mildew effector evades recognition by polymorphism of expression and subcellular localization. Nat. Commun. 9, 5192 (2018).

35. Tornero, P., Chao, R. A., Luthin, W. N., Goff, S. A. \& Dangl, J. L. Large-scale structurefunction analysis of the Arabidopsis RPM1 disease resistance protein. Plant Cell 14, 435-450 (2002).

36. Tsuda, K. et al. Dual regulation of gene expression mediated by extended MAPK activation and salicylic acid contributes to robust innate immunity in Arabidopsis thaliana. PLoS Genet. 9, e1004015 (2013).

37. Qi, D. et al. Recognition of the protein kinase AVRPPHB SUSCEPTIBLE1 by the disease resistance protein RESISTANCE TO PSEUDOMONAS SYRINGAE5 is dependent on s- 
acylation and an exposed loop in AVRPPHB SUSCEPTIBLE1. Plant Physiol. 164, 340-351 (2014).

38. Livak, K. J. \& Schmittgen, T. D. Analysis of relative gene expression data using real-time quantitative PCR and the 2(-Delta Delta C(T)) Method. Methods 25, 402-408 (2001).

39. Bray, N. L., Pimentel, H., Melsted, P. \& Pachter, L. Near-optimal probabilistic RNA-seq quantification. Nat. Biotechnol. 34, 525-527 (2016).

40. Guo, W. et al. 3D RNA-seq - a powerful and flexible tool for rapid and accurate differential expression and alternative splicing analysis of RNA-seq data for biologists. BioRxiv (2019). doi:10.1101/656686

41. Cho, H. K., Ahn, C. S., Lee, H.-S., Kim, J.-K. \& Pai, H.-S. Pescadillo plays an essential role in plant cell growth and survival by modulating ribosome biogenesis. Plant J. 76, 393-405 (2013).

42. Ingole, K. D., Dahale, S. K. \& Bhattacharjee, S. Proteomic analysis of SUMO1-SUMOylome changes during defense elicitation in Arabidopsis. BioRxiv (2020). doi:10.1101/2020.08.02.233544

43. Sohn, K. H., Zhang, Y. \& Jones, J. D. G. The Pseudomonas syringae effector protein, AvrRPS4, requires in planta processing and the KRVY domain to function. Plant J. 57, 10791091 (2009).

\section{Acknowledgments}

We thank Jeffery Dangl, Shuta Asai, Roger Innes, Kenichi Tsuda, Shuqun Zhang, Cyril Zipfel, Jian-Min Zhou, Albrecht von Arnim, Brian Staskawicz, Cecilia Cheval, Yasuhiro Kadota, Ruby O'Grady, Maddy Morris, Jack Rhodes and Yuli Ding for providing materials, discussion and technical support. We also thank Cyril Zipfel, Minhang Yuan, Xiufang Xin and Sheng-Yang He for critical reading of the manuscript. We thank the Gatsby Foundation (United Kingdom) for funding to the J.D.G.J. laboratory. B.P.M.N was supported by the Norwich Research Park Biosciences Doctoral Training Partnership from the Biotechnology and Biological Sciences Research Council (BBSRC) (grant agreement: BB/M011216/1). H.K.A. was supported by European Research Council Advanced Grant "ImmunitybyPairDesign" (grant agreement: 669926). P.D. acknowledges support from the European Union's Horizon 2020 Research and Innovation Program under Marie Skłodowska-Curie Actions (grant agreement: 656243) and a Future Leader Fellowship from BBSRC (grant agreement: BB/R012172/1). 


\section{Author contributions}

B.P.M.N., P.D. and J.D.G.J. conceived and conceptualized the study. B.P.M.N. performed the ROS assay, DAB staining, callose quantification, gene expression analysis, immunoblotting, protein serial dilution, RNA-seq, bacterial growth assay, HR assay and electrolyte leakage assay. B.P.M.N performed plasma membrane protein extraction with assistance from H.K.A.. H.K.A designed and performed the cycloheximide and MG132 experiment. H.K.A. and B.P.M.N. performed enrichment of ribosomes. P.D. performed the RNA-seq analyses. H.K.A. performed the statistical analyses. B.P.M.N. and P.D. wrote the original draft. B.P.M.N., H.K.A., P.D. and J.D.G.J. reviewed and edited the manuscript.

\section{Competing interest declaration}

The authors declare no competing interests.

\section{Data availability}

All data in this study are available within the article and Supplementary Information. RNA-seq data generated from this study are given in Supplementary Table 4. Statistical analyses of this study are provided in Supplementary Table 5. The original sequence data of RNA-seq that support the findings of this study have been deposited and made publicly available in the European Nucleotide Archive (ENA) with the primary accession code "PRJEB34955". All original gel blots can be found in the Supplementary Figure 1. Source Data from Fig. 1-4 and Extended Data Fig. 1-9 are provided with the paper.

\section{Correspondence and requests for materials should be addressed to P.D. or J.D.G.J.}

\section{Additional Information}

Supplementary Information is available for this paper.

Extended Data Fig. 1 | ETI ${ }^{\text {AvrRps4 }}$ and ETI ${ }^{\text {AvrRpt2 }}$ potentiates PTI responses. (a) Estradiol pretreatment in est:AvrRps4 leads to stronger and prolonged ROS burst compared to mock pretreatment. $\mathrm{n}=40$ leaf disks. (b) ROS accumulation over 55 mins in ETI ${ }^{\text {AvrRps4 }}$-pretreated leaves is significantly higher than mock-pretreated leaves. $n=120$ leaves over 3 independent experiments. (c) Pre-treatment of estradiol in est:AvrRps4 eds $1-2$ does not lead to stronger and prolonged ROS burst compared to mock pre-treatment. $\mathrm{n}=40$ leaf disks. (d) ROS accumulation over 55 mins in 
611 ETI ${ }^{\mathrm{AvrRps}^{4}}$-pretreated leaves in the eds1-2 is comparable to mock-pretreated leaves. $n=120$ leaves

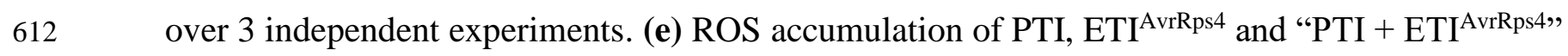
613 treated leaves during Phase I (0-60 mins), Phase II (60-300 mins) and Phase III (300-960 mins). $n$ $614=120$ leaves over 3 independent experiments.. (f) Summary table of ROS accumulation in different 615 phases. (g) "PTI + ETI $^{\text {AvrRps4" }}$ leads to a stronger PER4, WRKY31 transcript accumulation

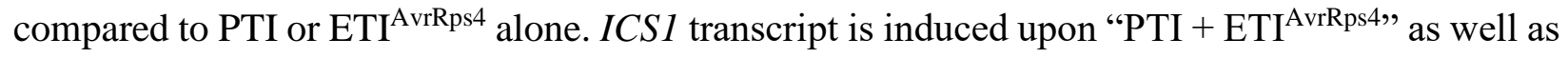
ETI $^{\text {AvrRps4 }}$ alone. Data points from 3 independent experiments were plotted onto the graphs, with \pm S.E. for error bars. Two-sided Welch's t-test was used to analyze significance differences between PTI $+\mathrm{ETI}^{\mathrm{AvrRps} 4}$ and PTI or ETI ${ }^{\mathrm{AvrRps} 4}(*, \mathrm{P} \leq 0.05 ; * *, \mathrm{P} \leq 0.01$; ***, P $\leq 0.005 ; * * * *, \mathrm{P}$ $\leq 0.001$; otherwise, not significant). Exact $\mathrm{P}$ values can be found in Supplementary Table 5. (h) PTI + ETI $^{\text {AvrRpt2 }}$ leads to prolonged ROS production during Phase II. $n=40$ leaf disks. (i) ROS accumulation of PTI, ETI ${ }^{\text {AvrRpt2 }}$ and PTI+ETI ${ }^{\text {AvrRpt2 }}$ treated leaves during Phase I, Phase II and Phase III and in total. $n=120$ leaves over 3 independent experiments. (j) Summary table of ROS accumulation in different phases. For (a), (c), (h) Solid line represents mean \pm S.E. (shaded curve) from one biological replicate. For (b), (d), (e), (i), data points from 3 independent experiments were analyzed with one-sided Kruskalis-Wallis test followed by post hoc Dunn's test. Different letters next to the boxplot indicate significant differences of $\mathrm{P}<0.05$. Centre lines represent the medians; bounds of box indicate the $25^{\text {th }}$ and $75^{\text {th }}$ percentiles; whiskers represent $1.5 \times$ interquartile range from $25^{\text {th }}$ and $75^{\text {th }}$ percentiles. $\mathrm{P}$-values were adjusted using Holm correction, and exact $\mathrm{P}$ values can be found in Supplementary Table 5. All experiments were repeated at least three times with similar results.

\section{Extended Data Fig. 2 | ETI ${ }^{\text {AvrRps4 }}$ enhances ROS production triggered by different PAMPs} and DAMP. (a-c) elf18-triggered ROS production in the presence of ETI ${ }^{\mathrm{AvrRps} 4}$ is stronger than elf18 treatment alone. (d-f) pep1-triggered ROS production in the presence of ETI ${ }^{\mathrm{AvrRps} 4}$ is stronger than pep1 treatment alone. (g-i) C10:0-triggered ROS production in the presence of ETI ${ }^{\text {AvrRps } 4}$ is stronger than C10:0 treatment alone. (j-l) nlp20-triggered ROS production in the presence of ETI $^{\text {AvrRps4 }}$ is stronger than nlp20 treatment alone. (m-o) Chitin-triggered ROS production in the presence of ETI ${ }^{\mathrm{AvrRps} 4}$ is stronger than chitin treatment alone. Shaded curves in (a), (d), (g), (j), (m) represent standard error (S.E.) and solid line represents mean.. $n=40$ leaf disks. ROS production in Phase I, Phase II, Phase III and total are shown as boxplots in (b), (e), (h), (k) and (n). Centre lines represent the medians; bounds of box indicate the $25^{\text {th }}$ and $75^{\text {th }}$ percentiles; 
whiskers represent $1.5 \times$ interquartile range from $25^{\text {th }}$ and $75^{\text {th }}$ percentiles. Data points from 3 independent experiments were analyzed with one-sided Kruskalis-Wallis test followed by post hoc Dunn's test. Different letters next to the boxplot indicate significant differences of $\mathrm{P}<0.05 . n=$ 120 leaves over 3 independent experiments. P values were adjusted using Holm correction, and exact $\mathrm{P}$ values can be found in Supplementary Table 5. (c), (f), (i), (l), (o) Tabular summary of total ROS production in different phases upon different PAMPs or DAMP treatments with ETI $^{\text {AvrRps4 }}$ co-activation. All experiments were repeated at least three times with similar results.

\section{Extended Data Fig. 3 | Protein accumulation of PTI signaling components during ETI. (a)} PTI signaling pathway. (b-c) Schematic representation of "natural infection mimicking" and "ETI pre-activation" experimental design. ETI ${ }^{\text {AvrRps4 }}$ was activated by estradiol treatment. * indicates activated immune system. (red: PTI activation; yellow: ETI activation, blue: PTI and ETI coactivation). (d) Pre-activation of ETI ${ }^{\text {AvrRps4 }}$ leads to accumulation and prolonged phosphorylation of MPK3 compared to mock pre-treatment. (e) Pre-activation of ETI ${ }^{\text {AvrRps4 }}$ leads to accumulation and prolonged phosphorylation of BIK1 and RbohD (S39 and S343) compared to mock pretreatment. Microsomal fractions from each sample were isolated for immunoblotting. Molecular weight marker (in $\mathrm{kDa}$ ) is indicated on the left. Ponceau staining (PS) was used as loading control. (f) Transcript induction of corresponding effectors and ICSI upon induced expression of AvrRpmI (dex:AvrRpm1), AvrRpt2 (est:AvrRpt2), AvrPphB (est:AvrPphB), AvrRps4 (est:AvrRps4) and AvrRpp4 (est:AvrRpp4). Extracted RNA were analyzed by qPCR and expression level is presented as relative to EFI $\alpha$. Data points from 3 independent experiments were plotted onto the graphs, with \pm S.E. for error bars.. Two-sided Welch's t-test was used to analyze significance in differences

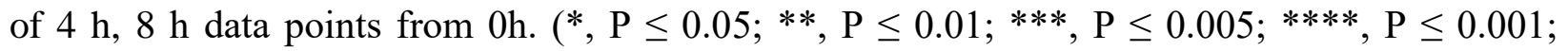
otherwise, not significant). Exact P-values can be found in Supplementary Table 5. (g) Protein accumulation of BAK1, SOBIR1, BIK1, RbohD, MPK3, MPK6, FLS2, CERK1 and MPK4 upon ETI activation for $4 \mathrm{~h}, 8 \mathrm{~h}$ in multiple effector inducible lines. 5-week-old leaves of inducible-

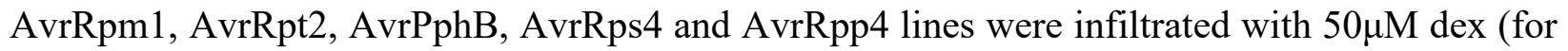
dex:AvrRpm1) or $50 \mu \mathrm{M}$ est. Samples were collected at 0,4 and $8 \mathrm{~h}$ post infiltration (hpi) for protein extraction. Molecular weight marker (in $\mathrm{kDa}$ ) is indicated on the left. Ponceau staining 
(PS) were used as loading control. All experiments were repeated at least three times with similar results.

\section{Extended Data Fig. 4 | Transcript accumulation of PTI signaling components during ETI.} (a) Relative gene expression of BAK1, SOBIR1, BIK1, RbohD, MPK3, MPK6, FLS2, CERK1, MPK4 and RbohF relative to EFla in multiple effector-inducible lines. 5-week-old leaves of inducible-AvrRpm1, AvrRpt2, AvrPphB, AvrRps4 and AvrRpp4 lines were infiltrated with $50 \mu \mathrm{M}$ dex (for dex:AvrRpm1) or $50 \mu \mathrm{M}$ est. Samples were collected at 0, 4 and 8 hpi for RNA extraction. (b) Heatmap of fold-changes $\left(\log _{2} \mathrm{FC}\right)$ of BAK1, SOBIR1, BIK1, RbohD, MPK3, MPK6, FLS2, CERK1, MPK 4 and RbohF from (a). Gene expression at $4 \mathrm{~h}$ and $8 \mathrm{~h}$ was normalized to expression level at $0 \mathrm{~h}$. Red indicates upregulation and blue indicates downregulation. (c) Protein accumulation of BIK1, RbohD, and MPK3 during ETI ${ }^{\mathrm{AvrRps} 4}$ is abrogated in eds 1-2. Proteins were extracted from est:AvrRps4 and est:AvrRps4 edsl-2 upon est treatment for $0 \mathrm{~h}, 4 \mathrm{~h}$, and $8 \mathrm{~h}$. Molecular weight marker (in $\mathrm{kDa}$ ) is indicated on the left. Ponceau staining (PS) were used as loading control. (d) Transcript induction of BIK1, RbohD, and MPK3 during ETI ${ }^{\mathrm{AvrRps} 4}$ is abrogated in $e d s 1-2$. For (a) and (d), extracted RNA were analyzed by qPCR and expression level is presented as relative to EF $1 \alpha$. Data points from 3 independent experiments were plotted onto the graphs, with \pm S.E. for error bars. Two-sided Welch's t-test was used to analyze significance in differences of $4 \mathrm{~h}, 8 \mathrm{~h}$ data points from $0 \mathrm{~h}(*, \mathrm{P} \leq 0.05 ; * *, \mathrm{P} \leq 0.01 ; * * *, \mathrm{P} \leq 0.005 ; * * * *, \mathrm{P} \leq$ 0.001; otherwise, not significant). Exact P-values can be found in Supplementary Table 5. All experiments were repeated at least three times with similar results.

Extended Data Fig. 5 | Genome-wide gene expression profiling of ETI ${ }^{\text {AvrRps4 }}$. (a) Schematic design of RNA-seq analysis. 5-week-old inducible lines of wild-type AvrRps4 (est:AvrRps4) and mutant AvrRps4 (estradiol-inducible AvrRps4 ${ }^{\text {KRVY135-138AAAA }}$-expressing line or est:AvrRps4 ${ }^{\text {mut }}$ ) were infiltrated with mock or $50 \mu \mathrm{M}$ est and samples were collected at $0 \mathrm{~h}$, and $4 \mathrm{~h}$. Samples from three biological replicates were collected for RNA-seq analysis. (b) 2573 differentially expressed (DE) genes were identified as significant in comparison between est:AvrRps4 treated with estradiol for $0 \mathrm{~h}$ (est:AvrRps4, Est-0h) and est:AvrRps4 treated with est for $4 \mathrm{~h}$ (est:AvrRps4, Est4h). P values for differentially expressed (DE) genes were generated with Fisher Z-transformation after Student's $t$-test. DE genes with "Benjamini and Hochberg's $(\mathrm{BH})$ method" false discovery rate $($ FDR) two-sided adjusted $\mathrm{P}$-value (adj.pval) $<0.01$ are categorized as significant. Heatmap 
representing the 2573 DE genes during 5 treatments; est:AvrRps4 (Untreated), est:AvrRps4 treated with est for $0 \mathrm{~h}$ (Est-0h), est:AvrRps 4 treated with est for $4 \mathrm{~h}$ (Est-4h), est:AvrRps ${ }^{\text {mut }}$ treated with est for $0 \mathrm{~h}$ (Est-0h) and est:AvrRps4 ${ }^{\text {mut }}$ treated with est for $4 \mathrm{~h}$ (Est-4h). Genes that are specifically upregulated during ETI ${ }^{\mathrm{AvrRps} 4}$ are in cluster 7 and 8. (c-e) GO enrichment analysis of genes from cluster 7 and 8. (c) Top three significantly enriched biological process GO-terms in cluster 7 and 8. (d) Top four significantly enriched molecular function GO-terms in cluster 7 and 8. (e) Top four significantly enriched cellular component GO-terms in cluster 7 and 8. For details of GO enrichment analysis refer to Source Data. (f) Red (positive $\log _{2} \mathrm{FC}$ (fold change)) represents genes that are significantly induced and blue (negative $\log _{2} \mathrm{FC}$ ) represents genes that are significantly repressed. BH-FDR two-sided adjusted P-value (adj.pval) $<0.05$ is considered as significant. Gradient of green color indicates significance of the adjusted P-value. For full list of DE genes refer to Supplementary Table 4.

\section{Extended Data Fig. 6 | Expression dynamics of PTI signaling components during ETI ${ }^{\text {AvrRps4. }}$}

(a) Transcript induction of SOBIR1, BAK1, BIK1, RbohD, MPK3, CERK1, MPK4, MPK6, ICS1, PR1 during ETI ${ }^{\text {AvrRps4 }}$ over 24 h. Transcript levels were normalized to EFl $\alpha$. Data points from 3 independent experiments were plotted onto the graphs, with \pm S.E. for error bars. Two-sided Welch's t-test was used to analyze significance in differences of data points from ETI ${ }^{\text {AvrRps4 }}{ }^{\text {- }}$ activated samples compared to untreated (UNT) samples (*, P $\leq 0.05 ; * *, P \leq 0.01 ; * * *, \mathrm{P} \leq 0.005$; ****, $\mathrm{P} \leq 0.001$; otherwise, not significant). Exact $\mathrm{P}$ values can be found in Supplementary Table 5. (b) Relative mRNA expression changes of ICS1 (green) and PRI (black) during ETI ${ }^{\text {AvrRps4 }}{ }^{\text {. }}$ Relative expression changes of the corresponding genes to untreated samples $\left(\log _{2} \mathrm{FC}=0\right.$, dotted line) are shown. Solid line represents mean \pm S.E. (shaded band). (c) Heatmap representing foldchanges $\left(\log _{2} \mathrm{FC}\right)$ of transcripts from (a). Gene expression at indicated time points are relative value to untreated samples. Red indicates upregulation and blue indicates downregulation. (d) Protein accumulation of PR1 at different time points. Ponceau staining of western blots from Fig. $3 \mathrm{~b}$ are also shown. (e) Serial dilution to estimate protein accumulation of BIK1, RbohD and MPK3 at $8 \mathrm{~h}$ after $\mathrm{ETI}^{\text {AvrRps } 4}$ activation compared to $0 \mathrm{~h}$. Red asterisk indicates approximate fold differences between $0 \mathrm{~h}$ and $8 \mathrm{~h}$. (f) 5-week old Arabidopsis rosette leaves of est:AvrRps4 were treated with $h r c C^{-}$, est, or " $h r c C^{-}+$est" for indicated timepoints and both RNA and proteins were extracted. Extracted RNA were analyzed by qPCR and expression level is presented as relative to EFl $\alpha$. Data points from 3 independent experiments were plotted onto the graphs, with \pm S.E. for 


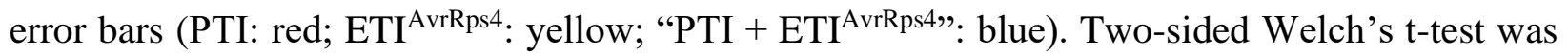
used to analyze significance in differences of $4 \mathrm{~h}, 8 \mathrm{~h}$ data points from $0 \mathrm{~h}(*, \mathrm{P} \leq 0.05 ; * *, \mathrm{P} \leq 0.01$; $* * *, \mathrm{P} \leq 0.005$; ****, $\mathrm{P} \leq 0.001$; otherwise, not significant). Exact $\mathrm{P}$-values can be found in Supplementary Table 5. For (d), (e), (f), Ponceau staining (PS) was used as loading control. Molecular weight marker (in $\mathrm{kDa}$ ) is indicated on the left. All experiments were repeated at least three times with similar results.

\section{Extended Data Fig. 7 | Multiple mechanisms are involved in the upregulation of PTI signaling} components during ETI ${ }^{\text {AvrRss4 }}$. (a) Relative gene expression of ICS1, BIK1, RbohD, MPK3 and MPK6 in seedlings pre-activated with ETI ${ }^{\text {AvrRps } 4}$ for $3 \mathrm{~h}$ prior to treatment with cycloheximide (CHX) and MG132. Data points from 3 independent experiments were plotted onto the graphs, with \pm S.E. for error bars.. Two-sided Welch's t-test was used to analyze significance in differences at $3 \mathrm{~h}$ compared to $0 \mathrm{~h}(*, \mathrm{P} \leq 0.05 ; * *, \mathrm{P} \leq 0.01 ; * * *, \mathrm{P} \leq 0.005 ; * * * *, \mathrm{P} \leq 0.001$; otherwise, not significant). Exact $\mathrm{P}$ values can be found in Supplementary Table 5. (b, c) Protein accumulation of MPK3, RbohD, BIK1, MPK6, Actin in seedlings pre-treated with Mock (DMSO) for $3 \mathrm{~h}$ (b), and RPS4-HA, FLS2, and BAK1 (c) in seedlings pre-treated with Mock or est, subsequently treated with CHX $(50 \mu \mathrm{M})$, MG132 $(10 \mu \mathrm{M})$, or both for indicated times $(2 \mathrm{~h}, 4 \mathrm{~h}, 8 \mathrm{~h})$. Actin was used as loading control. Ponceau staining (PS) of corresponding blots are shown below. For FLS2 and Actin, as well as BAK1 and BIK1, immunoblot was performed with membranes cut in half (above $70 \mathrm{kDa}$ for FLS2, BAK1, respectively, below $70 \mathrm{kDa}$ for Actin and BIK1 immunoblot, respectively). Therefore, Ponceau staining (PS) for FLS2 and Actin, BAK1 and BIK1, respectively, are identical. (d) Schematic representation of ribosome enrichment. (e-f) Ribosome was enriched, and (e) total extract (T), supernatant (S), and ribosomal pellet (P) samples were blotted with RPS6 and RPL10 antibody. For (b), (c) and (e), Ponceau staining (PS) was used as loading control. Molecular weight marker (in $\mathrm{kDa}$ ) is indicated on the left. (f) RNA extracted from total extract (Total RNA), and ribosomal pellet (Ribosome RNA) from mock and est-treated est:AvrRps4 samples were loaded on an agarose gel. 28S and 18S rRNA are indicated. (g) Relative expression of ICS1, SOBIR1, BAK1, BIK1, RbohD and MPK3 to EF1 $\alpha$ from total RNA (Total) and ribosomal pellet (Ribosomal). Data points from 3 independent experiments were plotted onto the graphs, with \pm S.E. for error bars. Two-sided Welch's t-test was used to analyze significance in differences of $6 \mathrm{~h}$ compared to $0 \mathrm{~h}(*, \mathrm{P} \leq 0.05 ; * *, \mathrm{P} \leq 0.01 ; * * *, \mathrm{P} \leq 0.005 ; * * * *, \mathrm{P} \leq 0.001$; otherwise, not significant). Exact P-values can be found in Supplementary Table 5. (h) Ratio of ribosomal RNA 
to total RNA (relative to $E F 1 \alpha$ ) of ICS1,SOBIR1, BAK1, BIK1, RbohD and MPK3 in mock and ETI samples. Values are calculated from the transcripts retained in the ribosomal samples over total samples. Data points from 3 independent experiments were plotted onto the graphs, with \pm S.E. for error bars. All experiments were repeated at least three times with similar results. Twosided Welch's t-test was used to analyze significance in differences of the translation efficiency (T.E.) between Mock and ETI-treated samples (* $\mathrm{P} \leq 0.05 ; * *, \mathrm{P} \leq 0.01 ; * * *, \mathrm{P} \leq 0.005 ; * * * *, \mathrm{P}$ $\leq 0.001$; otherwise, not significant). Exact P-values can be found in Supplementary Table 5 .

Extended Data Fig. 8 | ETI functions through PTI. (a) 5-week-old leaves of est:AvrRps4 were infiltrated with Pst strain DC3000 hrc $C^{-}$(Pst hrcC"; triggers PTI), Pst DC3000 (Pst; triggers "PTI

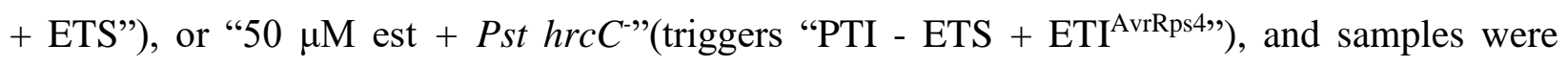
collected at the indicated time points for protein extraction and immunoblotting. PTI leads to activation of MAPKs and accumulation of BIK1 and RbohD (red). Pst secretes effectors to block PTI (green). Co-activation of PTI and ETI ${ }^{\text {AvrRps4 }}$ leads to stronger accumulation of MPK3, BIK1 and RbohD compared to PTI (blue). MAPKs activation is also prolonged during "PTI +

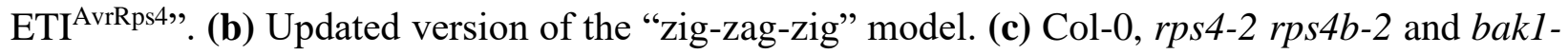
5 bkk1-1 were infected with Pst DC3000 carrying AvrRps4 (red) or empty vector (grey). Bacterial growth at 0 dpi as measured. $n=12$ leaves. (d) Col-0, rps 4-2 rps $4 b-2$ and fls 2 efr were infected with Pst DC3000 carrying AvrRps4 (red) or empty vector (grey). Both rps4-2 rps4b-2 (No ETI) and fls2 efr (PTI-reduced) are insufficient to provide resistance against Pst DC3000:AvrRps4 compared to Col-0 ("PTI + ETI"). Day 0: $n=12$ leaves; day 3: $n=18$ leaves. For (c), (d), data points were analyzed by one-way ANOVA followed by post hoc Tukey's HSD test. Data points with different letters indicate significant differences of $\mathrm{P}<0.01$. (e) flg22-induced ROS burst is not affected in rps4-2 rps4b-2. Shaded curve represents standard error (S.E.) and solid line represents average value from 24 leaves in each treatment during $n=24$ leaves . (f) flg22-induced ROS production over 55 mins in Col-0 and rps4-2 rps $4 b$-2. Data points from 3 biological replicates were analyzed with one-sided Kruskal-Wallis test followed by post hoc Dunn's test. Data points with different letters indicate significant differences of $\mathrm{P}<0.05 . n=72$ leaves over 3 independent experiments. (g) flg22-induced MPK phosphorylation is not affected in rps4-2 rps4b-2. Upon flg22 treatment, samples were taken at indicated time points for immunoblotting. For (a), (g), Ponceau staining (PS) was used as loading control. Molecular weight marker (in kDa) is indicated on the left. All experiments were repeated at least three times with similar results. For (c), (d), (f), 
centre lines represent the medians; bounds of box indicate the $25^{\text {th }}$ and $75^{\text {th }}$ percentiles; whiskers represent $1.5 \times$ interquartile range from $25^{\text {th }}$ and $75^{\text {th }}$ percentiles. Exact P-values can be found in Supplementary Table 5.

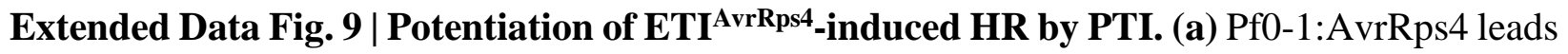
to macroscopic HR in est:AvrRps4 leaves. Both PTI (Pf0-1:AvrRps4 ${ }^{\text {mut }}$ ) or ETI ${ }^{\text {AvrRps4 }}$ (est) does not lead to macroscopic HR. Coactivation of PTI and ETI ${ }^{\text {AvrRps4 }}$ (est + Pf0-1:AvrRps4 ${ }^{\text {mut }}$ ) leads to macroscopic HR. The numbers indicate number of leaves displaying HR of the total number of leaves infiltrated. $n=18$ leaves. (b) Est:AvrRps4 leaves were hand-infiltrated with indicated solutions and electrolyte leakage was measured over 48hpi. Combination of "PTI + ETI AvrRps4" (blue dots, "est + Pf0-1:AvrRps4 ${ }^{\text {mut" }) ~ l e a d s ~ t o ~ s t r o n g e r ~ e l e c t r o l y t e ~ l e a k a g e ~ c o m p a r e d ~ t o ~ E T I ~}{ }^{\text {AvrRps4 }}$ (est) or PTI (Pf0-1:AvrRps4 ${ }^{\text {mut }}$ ) alone. Pf0-1:AvrRps4 (green) acts as a positive control. Data points from 3 biological replicates were analyzed with one-way ANOVA followed by post hoc Tukey's HSD test. Data point from each biological replicate is indicated with different shapes. Data points with different letters indicate $\mathrm{P}<0.01 . n=9$ data points; each represents data from 15 leaf discs. Exact P-values can be found in Supplementary Table 5. (c) PTI induced by flg22, elf18, pep1, C10:0, nlp20 or chitin does not lead to macroscopic HR. Coactivation of PTI (trigger by these PAMPs or DAMP) with ETI ${ }^{\mathrm{AvrRps} 4}$ leads to macroscopic HR. The numbers indicate number of leaves displaying HR of the total number of leaves infiltrated. $n=18$ leaves. (d) 5-week-old inducible AvrRpm1 (dex:AvrRpm1), AvrRpt2 (est:AvrRpt2), AvrPphB (est:AvrPphB), AvrRps4 (est:AvrRps4) and AvrRpp4 (est:AvrRpp4) Arabidopsis leaves were infiltrated with either dex (for dex:AvrRpm1 only) or est. All pictures were taken at 3 dpi. The numbers indicate the number of leaves displaying HR of the total number of leaves infiltrated. $n=18$ leaves. (e) Combination of "PTI + ETI" leads to stronger macroscopic HR in inducible-AvrRpm1, AvrRpt2, AvrPphB and AvrRpp4 Arabidopsis lines. All pictures were taken 3 dpi. The numbers indicate number of leaves displaying HR of the total number of leaves infiltrated. $n=18$ leaves. All experiments were repeated at least three times with similar results.

\section{Extended Data Fig. 10 | MAPKs and NADPH oxidases are involved in HR induced by PTI +} ETI. (a) MPK phosphorylation during ETI triggered by multiple effectors. Seedlings of dex:AvrRpm1, est:AvrRpt2, est:AvrPphB and est:AvrRpp4 lines were soaked in dex or est, solution respectively for indicated time points (dark yellow). Untreated (UNT) seedlings were used 
as negative control, seedlings treated with $100 \mathrm{nM}$ flg22 for $15 \mathrm{~min}$ (red, flg22) were used as positive control. (b) RbohD phosphorylation during ETI triggered by multiple effectors. Seedlings of dex:AvrRpm1, est:AvrRpt2, est:AvrPphB and est:AvrRpp4 were soaked in either mock (black), dex or est solution (dark yellow) for $6 \mathrm{~h}$. Microsomal fraction from seedlings were isolated for immunoblotting. For (a), (b), Ponceau staining (PS) was used as loading control. Molecular weight marker (in $\mathrm{kDa}$ ) is indicated on the left. (c) MPK6SR\#58 (mpk3 mpk6 $\mathrm{P}_{\mathrm{MPK}}: \mathrm{MPK6}{ }^{\mathrm{YG}}$ ) is a conditional $m p k 3 m p k 6$ double mutant. MPK6 ${ }^{\mathrm{YG}}$ has a larger ATP binding pocket than MPK6 ${ }^{\mathrm{WT}}$ and is sensitive to the inhibitor 1-Naphthyl-PP1 (NA-PP1, ATP analog). Pre-treatment with NAPP1 inhibits MPK6 ${ }^{\mathrm{YG}}$ and temporarily generates a mpk3 mpk6 double mutant. Both Col-0 and MPK6SR\#58 leaves were pre-infiltrated with either 1\% DMSO (mock) or $10 \mu \mathrm{M}$ NA-PP1. After $3 \mathrm{~h}$, these leaves were infiltrated with either Pf0-1:empty vector (triggers PTI) or Pf0-1:AvrRps4

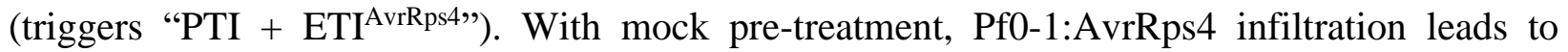
macroscopic HR in both Col-0 and MPKS6R\#58. NA-PP1 pre-treatment attenuates HR caused by Pf0-1:AvrRps4 only in the MPK6SR\#58 line. All pictures were taken at $1 \mathrm{dpi}$. The numbers indicate number of leaves displaying HR of the total number of leaves infiltrated. $n=18$ leaves. (d) Col-0 and rbohd rbohf leaves were infiltrated with either Pf0-1:empty vector (triggers PTI) or

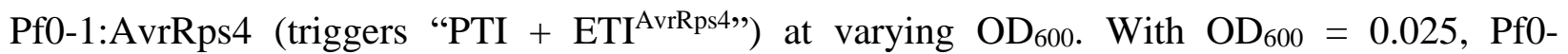
1:AvrRps4 infiltration leads to less macroscopic HR in rbohd rbohf. All pictures were taken 1 dpi. The numbers indicate number of leaves displaying HR of the total number of leaves infiltrated. $n$ $=18$ leaves. All experiments were repeated at least three times with similar results. (e) Model: Upon ligand detection by PRRs, PTI leads to activation of BIK1, RbohD and MAPKs. Activation of an NLR (ETI without PTI) elevates accumulation of PTI signaling components. Co-activation of both PTI and ETI elevates accumulation and enhances activation of multiple PTI signaling components, enabling a stronger immune response. 